\title{
ALMOST STATISTICAL CONVERGENCE IN AMENABLE SEMIGROUPS
}

\author{
FATIH NURAY and B. E. RHOADES*
}

\begin{abstract}
The purpose of this study is to extend the notion of almost statistical convergence to functions defined on discrete countable amenable semigroups.
\end{abstract}

\section{Introduction and Background}

Lorentz [9] defined the following characterization. A sequence $\left\{x_{n}\right\}$ is said to be almost convergent to $s$ if and only if

$$
\lim _{n \rightarrow \infty} \frac{1}{n} \sum_{k=1}^{n} x_{m+k}=s
$$

uniformly in $m$.

Let $G$ be a discrete countable amenable semigroup with identity in which both right and left cancelation laws hold, and $w(G)$ and $m(G)$ denote the spaces of all real valued functions and all bounded real functions $f$ on $G$, respectively. The space $m(G)$ is a Banach space with the supremum norm $\|f\|_{\infty}=\sup \{|f(g)|: g \in G\}$. A mean on $m(G)$ is a real linear functional $\varphi$ on $m(G)$ such that

$$
\inf \{f(g): g \in G\} \leq \varphi(f) \leq \sup \{f(g): g \in G\}
$$

for all $f \in m(G)$. Clearly $\varphi \geq 0$ and $\varphi(1)=1$, where 1 denotes the unity element on $G$. A mean $\varphi$ is said to be a left invariant mean if $\varphi(f(g h))=$ $\varphi(f(h))$ for all $g, h \in G$ and all $f \in m(G)$. There is the obvious analogous definition of a right invariant mean; $\varphi(f(h g))=\varphi(f(h))$. The semigroup $G$ is said to be left(right) amenable if there exists a left(right) invariant mean on $m(G)$ and $G$ is amenable if it is both left and right amenable. Let $G$ be amenable. A function $f \in m(G)$ is almost convergent to $s$ if $\varphi(f)=s$ for

\footnotetext{
* The first named author acknowledges the support of The Scientific and Technological Research Council of Turkey in the preparation of this work.

Received 7 April 2009, in final form 29 September 2011.
} 
every invariant left mean and every invariant right mean $\varphi$. (See, e.g., [3]) Namioka [12] showed that, if $G$ is a discrete countable amenable group, there exists a sequence $\left\{S_{n}\right\}$ of finite subsets of $G$ such that

(i) $G=\bigcup_{n=1}^{\infty} S_{n}$

(ii) $S_{n} \subset S_{n+1}, n=1,2,3, \ldots$, and

(iii) $\lim _{n \rightarrow \infty} \frac{\left|S_{n} g \cap S_{n}\right|}{\left|S_{n}\right|}=1, \lim _{n \rightarrow \infty} \frac{\left|g S_{n} \cap S_{n}\right|}{\left|S_{n}\right|}=1$ for all $g \in G$.

Here $|A|$ denotes the number of elements in the finite set $A$.

Any sequence of finite subsets of $G$ satisfying (i), (ii) and (iii) is called a Folner sequence for $G$.

The sequence $S_{n}=\{0,1,2, \ldots, n-1\}$ is a familiar Folner sequence giving rise to the classical Cesaro method of summability.

In [13] we have introduced the concepts of statistical convergent functions for any Folner sequence as follow:

Definition 1.1. Let $G$ be a discrete countable amenable semigroup with identity in which both right and left cancellation laws hold. An $f \in w(G)$ is said to be statistically convergent to $s$ for any Folner sequence $\left\{S_{n}\right\}$ for $G$ if, for every $\epsilon>0$,

$$
\lim _{n \rightarrow \infty} \frac{1}{\left|S_{n}\right|}\left|\left\{g \in S_{n}:|f(g)-s| \geq \epsilon\right\}\right|=0 .
$$

The set of all statistically convergent functions will be denoted by $S(G)$.

\section{Almost Statistical Convergence}

In [3] Douglas proved following theorem.

THEOREM 2.1. Let $G$ be a discrete countable amenable semigroup with identity in which both right and left cancelation laws hold. A necessary and sufficient condition that $f \in m(G)$ is almost convergent to $s$ is that, for any Folner sequence $\left\{S_{n}\right\}$ for $G$,

$$
\lim _{n \rightarrow \infty} \frac{1}{\left|S_{n}\right|} \sum_{g \in S_{n}} f(g h)=s \quad \text { and } \quad \lim _{n \rightarrow \infty} \frac{1}{\left|S_{n}\right|} \sum_{g \in S_{n}} f(h g)=s
$$

uniformly in $h$.

Now we define almost statistical convergence and almost statistically Cauchy function, for any Folner sequence $\left\{S_{n}\right\}$ for $G$. 
Definition 2.2. Let $G$ be a discrete countable amenable semigroup with identity in which both right and left cancelation laws hold. An $f \in w(G)$ is said to be almost statistically convergent to $s$, for any Folner sequence $\left\{S_{n}\right\}$ for $G$ if, for each $\epsilon>0$,

$$
\lim _{n \rightarrow \infty} \frac{1}{\left|S_{n}\right|}\left|\left\{g \in S_{n}:|f(g h)-s| \geq \epsilon\right\}\right|=0
$$

and

$$
\lim _{n \rightarrow \infty} \frac{1}{\left|S_{n}\right|}\left|\left\{g \in S_{n}:|f(h g)-s| \geq \epsilon\right\}\right|=0
$$

uniformly in $h$. The set of all almost statistically convergent functions will be denoted by $\hat{S}(G)$.

Definition 2.3. Let $G$ be a discrete countable amenable semigroup with identity in which both right and left cancelation laws hold. An $f \in w(G)$ is said to be almost statistically Cauchy, for any Folner sequence $\left\{S_{n}\right\}$ for $G$ if, for each $\epsilon>0$ there exists an $l(\epsilon) \in G$ such that

$$
\lim _{n \rightarrow \infty} \frac{1}{\left|S_{n}\right|}\left|\left\{g \in S_{n} h:|f(g)-f(l(\epsilon))| \geq \epsilon\right\}\right|=0
$$

and

$$
\lim _{n \rightarrow \infty} \frac{1}{\left|S_{n}\right|}\left|\left\{g \in h S_{n}:|f(g)-f(l(\epsilon))| \geq \epsilon\right\}\right|=0
$$

uniformly in $h$.

Also we define strongly almost convergent functions and $p$-strongly almost convergent functions for any Folner sequence $\left\{S_{n}\right\}$ for $G$.

Definition 2.4. Let $G$ be a discrete countable amenable semigroup with identity in which both right and left cancelation laws hold. We say that $f \in$ $m(G)$ is strongly almost convergent to $s$ if, for any Folner sequence $\left\{S_{n}\right\}$ for $G$,

$$
\lim _{n \rightarrow \infty} \frac{1}{\left|S_{n}\right|} \sum_{g \in S_{n}}|f(g h)-s|=0 \quad \text { and } \quad \lim _{n \rightarrow \infty} \frac{1}{\left|S_{n}\right|} \sum_{g \in S_{n}}|f(h g)-s|=0
$$

uniformly in $h$. The set of all strongly almost convergent functions will be denoted by $[\hat{c}(G)]$.

Definition 2.5. Let $G$ be a discrete countable amenable semigroup with identity in which both right and left cancelation laws hold and $0<p<\infty$. 
We say that $f \in m(G)$ is $p$-strongly almost convergent to $s$ if, for any Folner sequence $\left\{S_{n}\right\}$ for $G$,

$\lim _{n \rightarrow \infty} \frac{1}{\left|S_{n}\right|} \sum_{g \in S_{n}}|f(g h)-s|^{p}=0 \quad$ and $\quad \lim _{n \rightarrow \infty} \frac{1}{\left|S_{n}\right|} \sum_{g \in S_{n}}|f(h g)-s|^{p}=0$

uniformly in $h$. The set of all $p$-strongly almost convergent functions will be denoted by $[\hat{c}(G)]_{p}$.

THEOREM 2.6. Let $f \in w(G)$ and $p$ be a positive real number. Then

(a) $f$ is almost statistically convergent to $s$ for any Folner sequence $\left\{S_{n}\right\}$ for $G$ if it is p-strongly almost convergent to s for the same sequence,

(b) $[\hat{c}(G)]_{p}=\hat{S}(G) \cap m(G)$.

Since the proof of Theorem 2.6 is similar to that of Theorem 10 in [13], the proof is omitted.

The finite subsets $A_{1}, A_{2}, \ldots, A_{k} \subseteq G$ are called $\delta$-disjoint if there are subsets $B_{1}, B_{2}, \ldots, B_{k} \subseteq G$ such that

(i) $B_{i} \subseteq A_{i}, i=1,2, \ldots, k$,

(ii) $\frac{\left|B_{i}\right|}{\left|A_{i}\right|}>1-\delta$,

(iii) $B_{i} \cap B_{j}=\varnothing$ if $i \neq j \leq k$.

We say that the finite subsets $A_{1}, A_{2}, \ldots, A_{k} \subseteq G(1-\delta)$-cover a set $A \subseteq G$ if

$$
\frac{\left|A \cap\left(\bigcup_{i=1}^{k} A_{i}\right)\right|}{|A|} \geq 1-\delta \text {. }
$$

The finite subsets $A_{1}, A_{2}, \ldots, A_{k} \subseteq G \delta$-quasitile the set $A$, if there are finite sets $C_{1}, C_{2}, \ldots, C_{k} \subseteq G$ such that

(iv) $A_{i} C_{i} \subseteq A$ for any $i=1,2, \ldots, k$,

(v) $A_{i} C_{i} \cap A_{j} C_{j}=\emptyset$ if $i \neq j$,

(vi) $\left\{A_{i} c: c \in C_{i}\right\}$ form an $\delta$-disjoint family,

(vii) $\left\{A_{i} C_{i}: i=1,2, \ldots, k\right\}$ form a $(1-\delta)$ cover $A$.

The sets $C_{i}$ are called the tiling center (See, e.g., [5]).

The following result is due to Ornstein and Weiss [14] (see also [5]).

Lemma 2.7. Let $S_{1} \subseteq S_{2} \subseteq \ldots$ and $T_{1} \subseteq T_{2} \subseteq$. . be two Folner sequences. Let $\delta \in\left(0, \frac{1}{4}\right)$ and $\bar{N} \in \bar{N}$. Then there exist integers $n_{1}, n_{2}, \ldots, n_{k}$ with $N \leq n_{1}<n_{2}<\cdots<n_{k}$ such that $S_{n_{1}}, \ldots, S_{n_{k}} \delta$-quasitile $T_{m}$ when $m$ is large enough. 
THEOREM 2.8. The almost statistical convergence of the $f(g)$ does not depend on the particular choice of the Folner sequence.

Proof. Let the function $f(g)$ be almost statistically convergent to $s$ with respect to the Folner sequence $\left\{S_{n}\right\}$. We shall show that the function $f(g)$ is almost statistically convergent to $s$ with respect to the Folner sequence $\left\{T_{n}\right\}$ too. Since $f(g)$ is almost statistically convergent to $s$ with respect to the Folner sequence $\left\{S_{n}\right\}$ we can write for every $\epsilon>0$

$$
\lim _{n \rightarrow \infty} \frac{1}{\left|S_{n}\right|}\left|\left\{g \in S_{n}:|f(g h)-s| \geq \epsilon\right\}\right|=0
$$

uniformly in $h$. This means that

$$
\lim _{n \rightarrow \infty} \frac{1}{\left|S_{n}\right|} \sup _{h \in G}\left|\left\{g \in S_{n}:|f(g h)-s| \geq \epsilon\right\}\right|=0 .
$$

Let us consider the Folner sequence $\left\{T_{n}\right\}$. Let $\delta \in\left(0, \frac{1}{4}\right)$ and $N \in \mathrm{N}$. By Lemma 2.7, there exist integers $n_{1}, n_{2}, \ldots, n_{k}$ with $N \leq n_{1}<n_{2}<\cdots<n_{k}$ such that when $\mathrm{n}$ is large enough $S_{n_{1}}, \ldots, S_{n_{k}} \delta$-quasitile $T_{n}$ with tiling center $C_{1}^{n}, C_{2}^{n}, \ldots, C_{k}^{n}$. Thus when $n$ is large enough then $T_{n} \supseteq \bigcup_{i=1}^{k} S_{n_{i}} C_{i}^{n}$ and $\left|\bigcup_{i=1}^{k} S_{n_{i}} C_{i}^{n}\right| \geq \max \left\{(1-\delta)\left|T_{n}\right|,(1-\delta) \sum_{i=1}^{k}\left|C_{i}^{n}\right|\left|S_{n_{i}}\right|\right\}$ which implies

$$
\begin{aligned}
\frac{1}{\left|T_{n}\right|} \sup _{h \in G}\left|\left\{g \in T_{n}:|f(g h)-s| \geq \epsilon\right\}\right| & \\
\leq & \frac{1}{\left|T_{n}\right|} \sup _{h \in G}\left|\left\{g \in T_{n} \backslash \bigcup_{i=1}^{k} S_{n_{i}} C_{i}^{n}:|f(g h)-s| \geq \epsilon\right\}\right| \\
& +\frac{1}{\left|T_{n}\right|} \sup _{h \in G}\left|\left\{g \in \bigcup_{i=1}^{k} S_{n_{i}} C_{i}^{n}:|f(g h)-s| \geq \epsilon\right\}\right| \\
\leq & \frac{1}{\left|T_{n}\right|} \sup _{h \in G}\left|\left\{g \in T_{n} \backslash \bigcup_{i=1}^{k} S_{n_{i}} C_{i}^{n}:|f(g h)-s| \geq \epsilon\right\}\right| \\
& +\frac{1}{\left|\bigcup_{i=1}^{k} S_{n_{i}} C_{i}^{n}\right|} \sup _{h \in G}\left|\left\{g \in \bigcup_{i=1}^{k} S_{n_{i}} C_{i}^{n}:|f(g h)-s| \geq \epsilon\right\}\right| \\
\leq & +\frac{1}{\left|\bigcup_{i=1}^{k} S_{n_{i}} C_{i}^{n}\right|} \sup _{h \in G}\left|\left\{g \in \bigcup_{i=1}^{k} S_{n_{i}} C_{i}^{n}:|f(g h)-s| \geq \epsilon\right\}\right|
\end{aligned}
$$




$$
\begin{aligned}
& \leq \delta+\sum_{i=1}^{k} \frac{\left|C_{i}^{n}\right| \sup _{h \in G}\left|\left\{g \in S_{n_{i}}:|f(g h)-s| \geq \epsilon\right\}\right|}{(1-\delta) \sum_{i=1}^{k}\left|C_{i}^{n}\right|\left|S_{n_{i}}\right|} \\
& \leq \delta+\frac{1}{1-\delta} \max _{1 \leq i \leq k} \frac{\sup _{h \in G}\left|\left\{g \in S_{n_{i}}:|f(g h)-s| \geq \epsilon\right\}\right|}{\left|S_{n_{i}}\right|} \\
& \leq \delta+\frac{1}{1-\delta} \sup _{m \geq N} \frac{\sup _{h \in G}\left|\left\{g \in S_{m}:|f(g h)-s| \geq \epsilon\right\}\right|}{\left|S_{m}\right|} .
\end{aligned}
$$

Now letting $\delta \rightarrow 0^{+}$and $N \rightarrow+\infty$, we have

$$
\begin{aligned}
\frac{1}{\left|T_{n}\right|} \sup _{h \in G} \mid\left\{g \in T_{n}:|f(g h)-s|\right. & \geq \epsilon\} \mid \\
& \leq \frac{1}{\left|S_{n}\right|} \sup _{h \in G}\left|\left\{g \in S_{n}:|f(g h)-s| \geq \epsilon\right\}\right|
\end{aligned}
$$

and

$$
\lim _{n \rightarrow \infty} \frac{1}{\left|T_{n}\right|} \sup _{h \in G}\left|\left\{g \in S_{n}:|f(g h)-s| \geq \epsilon\right\}\right|=0 .
$$

Therefore

$$
\lim _{n \rightarrow \infty} \frac{1}{\left|T_{n}\right|}\left|\left\{g \in S_{n}:|f(g h)-s| \geq \epsilon\right\}\right|=0
$$

uniformly in $h$. A similar argument holds for the other limit.

\section{Almost Statistical Limit Points and Almost Statistical Cluster Points}

In [8] Fridy introduced the concepts of statistical limit point and statistical cluster point of sequences. In [13] we introduced the concepts of statistical limit point and statistical cluster point of functions defined on discrete countable amenable semigroups. In this section we introduce almost statistical limit point and almost statistical cluster point of functions defined on discrete countable amenable semigroups.

Definition 3.1. Let $S$ be a subset of $G$ and define

$$
\lim _{n \rightarrow \infty} \frac{1}{\left|S_{n}\right|}\left|\left\{g \in S_{n}: g h \in S\right\}\right|
$$

and

$$
\lim _{n \rightarrow \infty} \frac{1}{\left|S_{n}\right|}\left|\left\{g \in S_{n}: h g \in S\right\}\right|
$$

uniformly in $h$. When the above limits exist and are equal, we say that $S$ has almost Folner density, and this will be denoted by $\delta_{a}(S)$. If $f \in w(G)$ and 
$H \subset G$, we write $R_{f}(G)$ to denote the range of $f \in w(G)$. If $R_{f}(H)$ is a subset of $R_{f}(G)$ and

$$
\lim _{n \rightarrow \infty} \frac{1}{\left|S_{n}\right|}\left|\left\{g \in S_{n}: g h \in H\right\}\right|=0
$$

and

$$
\lim _{n \rightarrow \infty} \frac{1}{\left|S_{n}\right|}\left|\left\{g \in S_{n}: h g \in H\right\}\right|=0
$$

uniformly in $h$, then $R_{f}(H)$ is called a subset of almost Folner density zero for any Folner sequence $\left\{S_{n}\right\}$ for $\mathrm{G}$ or a almost thin subset. Otherwise we say that $R_{f}(H)$ is a almost nonthin subset of $R_{f}(G)$.

Definition 3.2. The number $s$ is an almost statistical limit point for an $f \in w(G)$, for any Folner sequence $\left\{S_{n}\right\}$ for $G$ provided that, there is an almost nonthin subset of $R_{f}(G)$ that $\mathrm{f}$ almost convergent to $s$ in it.

Definition 3.3. The number $c$ is an almost statistical cluster point for an $f \in w(G)$ provided that, for each $\epsilon>0$ the set $\left\{g \in S_{n}:|f(g)-c|<\epsilon\right\}$ does not have almost density zero for any Folner sequence $\left\{S_{n}\right\}$ for $G$.

\section{REFERENCES}

1. Connor, J. S., The statistical and strong p-Cesàro convergence of sequences, Analysis 8 (1988), 46-63.

2. Day, M., Amenable semigroups, Illinois J. Math. 1 (1957), 509-544.

3. Douglass, S. A., On a concept of summability in amenable semigroups, Math. Scand. 23 (1968), 96-102.

4. Douglass, S. A., Summing sequences for amenable semigroups, Michigan Math J. 20 (1973), 169-179.

5. Elek, G., The rank of finitely generated modules over group algebras, Proc. Amer. Math. Soc. 131 (2003), 3477-3485.

6. Fast, H., Sur la convergence statistique, Colloq. Math. 2 (1951), 241-244.

7. Fridy, J. A., On statistical convergence, Analysis 5 (1985), 301-313.

8. Fridy, J. A., Statistical limit points, Proc. Amer. Math. Soc. 118 (1993), 1187-1192.

9. Lorentz, G., A contribution to the theory of divergent sequences, Acta Math. 80 (1948), 167-190.

10. Mah, P. F., Summability in amenable semigroups, Trans. Amer. Math. Soc. 156 (1971), 391403.

11. Mah, P. F., Matrix summability in amenable semigroups, Proc. Amer. Math. Soc. 36 (1972), 414-420.

12. Namioka, I., Følner's conditions for amenable semigroups, Math. Scand. 15 (1964), 18-28.

13. Nuray, F., and Rhoades, B. E., Some kinds of convergence defined by Folner sequences, Analysis 31 (2011), 381-390.

14. Ornstein, D. S., and Weiss, B., Entropy and isomorphism theorems for actions of amenable groups, J. Analyse Math. 48 (1987), 1-141. 
15. Zygmund, A., Trigonometric Series, 2nd ed., Cambridge Univ. Press, Cambridge 1979.

DEPARTMENT OF MATHEMATICS AFYON KOCATEPE UNIVERSITY AFYONKARAHISAR

TURKEY

E-mail: fnuray@aku.edu.tr
INDIANA UNIVERSITY BLOOMINGTON, IN

USA

E-mail: rhoades@indiana.edu 